\title{
Highly Stable Super-Implicit Hybrid Methods for Special Second Order IVPs
}

\author{
Oluwasegun Micheal Ibrahim ${ }^{1,2}$, Monday Ndidi Oziegbe Ikhile ${ }^{2}$ \\ ${ }^{1}$ Department of Mathematical Sciences, African Institute for Mathematical Sciences, Kigali City, Rwanda \\ ${ }^{2}$ Advance Research Laboratory, Department of Mathematics, University of Benin, Benin City, Nigeria
}

\section{Email address:}

oluwasegun.micheal@aims.ac.rw (O. M. Ibrahim),mnoikhilo@yahoo.com (M. N. O. Ikhile)

\section{To cite this article:}

Oluwasegun Micheal Ibrahim, Monday Ndidi Oziegbe Ikhile. Highly Stable Super-Implicit Hybrid Methods for Special Second Order IVPs. American Journal of Applied Scientific Research. Vol. 3, No. 3, 2017, pp. 21-27. doi: 10.11648/j.ajasr.20170303.11

Received: June 29, 2017; Accepted: July 14, 2017; Published: October 31, 2017

\begin{abstract}
The idea of symmetric super-implicit linear multi-step methods (SSILMMs) necessitates the use of not just past and present solution values of the ordinary differential equations (ODEs), but also, future values of the solution. Such methods have been proposed recently for the numerical solution of second-order ODEs. One technique to obtain more accurate integration process is to construct linear multi-step methods with hybrid points employing future solution values. In this regard, we construct families of Störmer-Cowell type hybrid SSILMMs having higher order than that of the symmetric super-implicit method recently proposed for the same step number using the Taylors series approach. The newly derived hybrid SSILMMs are p-stable with accurate results when tested on some special second order IVPs.
\end{abstract}

Keywords: Super-Implicit, Hybrid LMM, Störmer-Cowell Method, P-stability

\section{Introduction}

Consider the initial value problem (IVP),

$$
y^{\prime \prime}(x)=f(x, y(x)) ; y\left(x_{0}\right)=y_{0}, y^{\prime}\left(x_{0}\right)=y_{0},
$$

in ordinary differential equations (ODEs) in which there is no explicit first derivative appearing. There is vast literature for the numerical solution of (1), see [13], [5], and references therein. The linear multi-step methods for solving the second order IVP (1) is,

$$
\sum_{j=0}^{k} \alpha_{j} y_{n+j}=h^{2} \sum_{j=0}^{k} \beta_{j} f_{n+j}, \beta_{k} \neq 0 .
$$

The first and second characteristic polynomials are,

$$
\rho(z)=\sum_{j=0}^{k} \alpha_{j} z^{j}, \sigma(z)=\sum_{j=0}^{k} \beta_{j} z^{j} .
$$

The LMM (2) has an associated local truncation error (LTE) difference operator,

$$
\begin{gathered}
L[y(x) ; h]=\sum_{j=0}^{k} \alpha_{j} y(x+j h)-h^{2} \sum_{j=0}^{k} \beta_{j} y^{\prime \prime(x+j h)}= \\
C_{p+2} h^{p+2} y^{(p+2)}\left(x_{n}\right)+O\left(h^{(p+3)}\right),
\end{gathered}
$$

where $C_{p+2} h^{p+2} y^{(p+2)}\left(x_{n}\right)$ is the LTE at the point $x_{n}, p$ is the order of the method, and $C_{p+2}$ is the error constant given by,

$$
\begin{aligned}
C_{q} & =\frac{1}{q !} \sum_{j=0}^{k} j^{q+2}\left(j^{2} \alpha_{j}-q(q-1) \beta_{j}\right) \\
& -\sum_{k+1=0}^{k} \frac{j^{q-2}}{(q-2) !} \beta_{j}, q>2 .
\end{aligned}
$$

As the usual convention, method (2) is assumed to satisfy the following conditions,

1. $\alpha_{k}=1,\left|\alpha_{0}\right|+\left|\beta_{0}\right| \neq 0$ (real parameters),

2. $\rho(z)$ and $\sigma(z)$ have no common factor (irreducibility),

3. $\rho(1)=\rho^{\prime}(1)=0, \rho^{\prime \prime}(1)=2 \sigma(1)$ (consistency),

4. zero-stable.

The method (2) is symmetric if $\alpha_{j}=\alpha_{k-j}$ and $\beta_{j}=\beta_{k-j}$ for $j=0(1) k$. The stability of method (2) is determined by the application on the periodic test problem,

$$
y^{\prime \prime}+\omega^{2} y=0, \omega, y \in \Re .
$$

Some preliminary definitions are given.

Definition 1 [14]: The LMM (2) is said to have an interval of periodicity $\left(0, H^{2}\right)$, if for all $H^{2}$ in this interval, the roots of, 


$$
P\left(z, H^{2}\right)=\rho(z)+H^{2} \sigma(z)=0, H=\omega h,
$$

satisfy, $\quad z_{1}=e^{i \theta(H)}, z_{2}=e^{-i \theta(H)},\left|z_{t}\right| \leq 1, t \geq 3, \ldots, k$, $\theta(H) \in \Re$.

Definition 2 [14]: The LMM (2) is said to be p-stable, if its interval of periodicity is $(0, \infty)$.

Definition 3 [18]: Method (2) is almost p-stable, if its interval of periodicity is $(0, \infty)-d$, where $d$ is a set of distinct points.

The result put forward by [13] have shown that no LMM (2) of order greater than $p=2$ can be $p$-stable. Also, [8] has proved to support [13]'s claim. Precisely, [8]'s result is stated.

Theorem 4 [8]: Consider an irreducible, convergent, symmetric multi-step method (2). Define the function,

$$
\eta(\theta)=\frac{-\rho\left(e^{i \theta}\right)}{\sigma\left(e^{i \theta}\right)}
$$

Then, the method (2) has a non-vanishing interval of periodicity if and only if,

(1) $\eta(\theta)$ has a non-zero double roots in the interval $\theta \in(0, \pi)$,

(2) $\eta^{\prime \prime}(\theta)$ is positive on all the non-zero double roots of $\eta(\theta)$ in interval $\theta \in(0, \pi)$.

Cash [1] independently showed that the order barrier on attainable order of a p-stable LMM (2) could be bypassed by considering certain hybrid two-step methods. An example of the method from this family is given by,

$$
\begin{gathered}
y_{n+2}-2 y_{n+1}+y_{n}=h^{2}\left(\frac{-11}{360}\left(f_{n+2}+f_{n}\right)+\frac{3}{20} f_{n+1}+\right. \\
\left.\frac{41}{90}\left(f_{n+\frac{3}{2}}+f_{n+\frac{1}{2}}\right)\right) .
\end{gathered}
$$

The order is $p=4, C_{P+2}=\frac{17}{5760}$, with hybrid pair,

$$
y_{n+\frac{3}{2}}=\frac{1}{4} y_{n+2}+y_{n+1}-\frac{1}{4} y_{n}+h^{2}\left(\frac{-3}{48} f_{n+2}+\frac{9}{48} f_{n+1}\right),
$$

and $y_{n+\frac{1}{2}}=\frac{1}{2} y_{n+1}+\frac{1}{2} y_{n}+h^{2}\left(\frac{9}{192} f_{n+2}-\frac{15}{96} f_{n+1}-\frac{1}{64} f_{n}\right)$.

The interval of periodicity of $(9)$ is $(0, \infty)$, and it is pstable.

As [1] further noted, [12] claimed to have derived high order p-stable linear multi-step methods but their concept of p-stability is considerably different from that given in [14]. The work in [2] further stressed on the work in [1], by considering the free parameters available in their proposed linear multi-step methods which can reduce the work to two functional evaluations, and also, reduces the work with respect to implementation for nonlinear problems of (1). Fatunla [4] derived a one-leg scheme found to be advantageous in terms of functions evaluations. Only one function evaluation and $k$ values of $y$ need to be stored for use in the next integration step. Fatunla et al [6] used the concept of Padé approximation to obtain a p-stable linear multi-step method,

$$
y_{n+2}-2 y_{n+1}+y_{n}=h^{2} f\left(x_{n+1}, \frac{1}{12} y_{n+2}+\frac{5}{6} y_{n+1}+\frac{1}{12} y_{n}+h^{2}\left(\frac{-1}{144} f_{n+2}+\frac{5}{72} f_{n+1}+\frac{-1}{144} f_{n}\right)\right),
$$

with order $p=4, C_{p+2}=\frac{17}{5760}$, which is an extension of the scheme in [4]. The concept of p-stability based on [14] (definition (2)) which was also employed in [1] and [6] will be adopted in this paper. Several methods based on LMM have been proposed see for example, [21], [22], [23], and [25]. Neta [16] considered a very special class of (2), the symmetric super-implicit linear multi-step method given by,

$$
\begin{gathered}
\sum_{j=0}^{\frac{k}{2}} \alpha_{j}\left(y_{n+j}+y_{n-j}\right)=h^{2} \sum_{j=0}^{\frac{k^{\prime}}{2}} \beta_{j}\left(f_{n+j}+f_{n-j}\right) . \quad(11) \quad 8, \\
y_{n+2}-2 y_{n+1}+2 y_{n}-2 y_{n-1}+y_{n-2}= \\
h^{2}\left(\frac{7411}{72576} f_{n}+\frac{362771}{453600}\left(f_{n+1}+f_{n-1}\right)+\frac{47057}{453600}\left(f_{n+2}+f_{n-2}\right)-\frac{2707}{453600}\left(f_{n+3}+f_{n-3}\right)+\right. \\
\left.\frac{641}{1814400}\left(f_{n+4}+f_{n-4}\right)\right), \mathrm{p}=10, C_{p+2}=\frac{-4139}{79833600} .
\end{gathered}
$$

However, the Taylors series approach in the sense of the work in [1] will be used to derive the new hybrid extension of (11) in [16] while using MATHEMATICA v 8 [11].

\section{Construction of Hybrid Symmetric Super-Implicit Obreckoff Type LMM}

The class of methods to be considered is in the general class of the hybrid method,

$$
\sum_{j=0}^{\frac{k}{2}} \psi_{j}\left(y_{n+j}+y_{n-j}\right)=\sum_{j=1}^{m} h^{2 l}\left(\sum_{j=0}^{\frac{s}{2}} \beta_{j}^{(l)}\left(f_{n+j}^{(2 i-2)}+f_{n-j}^{(2 i-2)}\right)\right)+\sum_{l=1}^{q} h^{2 l} \phi^{(l)}\left(f_{n+\lambda}{ }^{(2 i-2)}+f_{n-\lambda}^{(2 i-2)}\right) .
$$

This is an Obreckoff type class of methods, where the hybrids are given by, 


$$
\begin{gathered}
\sum_{j=0}^{\frac{k}{2}} \alpha_{j}\left(y_{n+j}+y_{n-j}\right)=\sum_{j=1}^{m} h^{2 l}\left(\sum_{j=0}^{\frac{s}{2}} \gamma_{j}^{(l)}\left(f_{n+j}{ }^{(2 i-2)}+f_{n-j}{ }^{(2 i-2)}\right)\right), \\
\sum_{j=0}^{\frac{k}{2}} a_{j}\left(y_{n+j}+y_{n-j}\right)=\sum_{j=1}^{m} h^{2 l}\left(\sum_{j=0}^{\frac{s}{2}} b_{j}{ }^{(l)}\left(f_{n+j}{ }^{(2 i-2)}+f_{n-j}{ }^{(2 i-2)}\right)\right) .
\end{gathered}
$$

In particular, is the hybrid SSILMM,

$$
\sum_{j=0}^{\frac{k}{2}} \psi_{j}\left(y_{n+j}+y_{n-j}\right)=h^{2} \sum_{j=0}^{\frac{s}{2}} \beta_{j}\left(f_{n+j}+f_{n-j}\right)++h^{2} \phi\left(f_{n+\lambda}+f_{n-\lambda}\right),
$$

when $m=1, q=1$ in (13), this is also considered in [15], where $k$ and the super-implicit parameter $s$ are even. The method (16) is explicit for $s=k-1$, implicit for $s=k$, and super-implicit for $s>k$ with $\lambda \in[0,1]$ as in [1]. Here the $\psi_{j}$ are fixed, say $\psi_{1}=1, \psi_{0}=-1$ to satisfy the zero-stability condition. The constants $(\beta)_{j=0(1) \frac{s}{2}}$ are then determined. The method (13) approximates the hybrid quantities $y_{n \pm \lambda}$ by an expression involving the quantities $\left\{\left(y_{n \pm j} ; f_{n \pm j}\right)\right\}$ only. For $m=1$ in (14) and (15), we have,

$$
\begin{gathered}
y_{n+\lambda}=\sum_{j=0}^{\frac{k}{2}} \alpha_{j}\left(y_{n+j}+y_{n-j}\right)+h^{2} \sum_{j=0}^{\frac{s}{2}} \gamma_{j}\left(f_{n+j}+f_{n-j}\right), \\
y_{n-\lambda}=\sum_{j=0}^{\frac{k}{2}} a_{j}\left(y_{n+j}+y_{n-j}\right)+h^{2} \sum_{j=0}^{\frac{s}{2}} b_{j}\left(f_{n+j}+f_{n-j}\right) .
\end{gathered}
$$

However, the hybrid of interest is

$$
\begin{aligned}
& y_{n+\lambda}=\sum_{j=0}^{k} \alpha_{j} y_{n-1+j}+h^{2} \sum_{j=0}^{\frac{s}{2}} \gamma_{j} f_{n+j}, \\
& y_{n-\lambda}=\sum_{j=0}^{k} a_{j} y_{n-1+j}+h^{2} \sum_{j=0}^{\frac{s}{2}} b_{j} f_{n-j},
\end{aligned}
$$

where, $k$ and the super-implicit parameter $s$ are even, and $\lambda \in[0,1]$ as in $[1]$.

\section{Construction of High Order Störmer-Cowell Type Hybrid SSILMMs}

This section presents the Störmer-Cowell type hybrid SSILMMs of order $p=10$, and $p=12$ respectively with hybrid parameter $\lambda$. When $k=2, s=6$, and $\psi_{j}$ are arbitrarily chosen as in section (2) and substituted into (16), we have,

$$
y_{n+1}-2 y_{n}+y_{n-1}=h^{2}\left(2 f_{n} \beta_{0}+\left(f_{n+1}+f_{n-1}\right) \beta_{1}+\left(f_{n+2}+f_{n-2}\right) \beta_{2}+\left(f_{n+3}+f_{n-3}\right) \beta_{3}+\emptyset\left(f_{n+\lambda}+f_{n-\lambda}\right)\right) \text {. }
$$

The following consistent simultaneous order condition are obtained as,

$$
\begin{gathered}
h^{0}:\left(1-2 \emptyset-2 \beta_{0}-2 \beta_{1}-2 \beta_{2}-2 \beta_{3}\right) \\
h^{2}: \frac{1}{12}\left(1-12 \lambda^{2} \emptyset-12 \beta_{1}-48 \beta_{2}-108 \beta_{3}\right) \\
h^{4}: \frac{1}{360}\left(1-30 \lambda^{4} \emptyset-30 \beta_{1}-480 \beta_{2}-2430 \beta_{3}\right) \\
h^{6}: \frac{\left(1-56 \lambda^{6} \emptyset-56 \beta_{1}-3584 \beta_{2}-40824 \beta_{3}\right)}{20160} \\
h^{8}: \frac{\left(1-90 \lambda^{8} \emptyset-90 \beta_{1}-23040 \beta_{2}-590490 \beta_{3}\right)}{1814400} .
\end{gathered}
$$

For hybrid parameter $\lambda=\frac{1}{2}$, method (21) become,

$y_{n+1}-2 y_{n}+y_{n-1}=h^{2}\left(\frac{20017}{90720} f_{n}+\frac{671}{36288}\left(f_{n+1}+f_{n-1}\right)-\frac{241}{2268000}\left(f_{n+2}+f_{n-2}\right)+\frac{13}{4536000}\left(f_{n+3}+f_{n-3}\right)+\frac{18496}{70875}\left(f_{n+\lambda}+f_{n-\lambda}\right)\right)$

with order $p=10$ and LTE $=\frac{-y^{(12)}[x] h^{12}}{25344000}$. For the hybrid,

$$
y_{n+\lambda}=\alpha_{2} y_{n+1}+\alpha_{1} y_{n}+\alpha_{0} y_{n-1}+h^{2}\left(\gamma_{0} f_{n}+\gamma_{1} f_{n+1}+\gamma_{2} f_{n+2}+\gamma_{3} f_{n+3}\right) .
$$

We obtain the consistent order equations,

$$
\begin{gathered}
\alpha_{0}=\frac{1}{114}\left(21 \lambda-60 \lambda^{3}+55 \lambda^{4}-18 \lambda^{5}+2 \lambda^{6}\right) \\
\alpha_{1}=\frac{1}{57}\left(57-78 \lambda+60 \lambda^{3}-55 \lambda^{4}+18 \lambda^{5}-2 \lambda^{6}\right)
\end{gathered}
$$

$$
\begin{gathered}
\alpha_{2}=\frac{1}{114}\left(135 \lambda-60 \lambda^{3}+55 \lambda^{4}-18 \lambda^{5}+2 \lambda^{6}\right) \\
\gamma_{0}=\frac{-3313 \lambda+3420 \lambda^{2}+2110 \lambda^{3}-3280 \lambda^{4}+1203 \lambda^{5}-140 \lambda^{6}}{6840}
\end{gathered}
$$




$$
\begin{array}{cc}
\gamma_{1}=\frac{-1641 \lambda+1920 \lambda^{3}-50 \lambda^{4}-279 \lambda^{5}+50 \lambda^{6}}{6840} & \gamma_{3}=\frac{-47 \lambda+80 \lambda^{3}-10 \lambda^{4}-33 \lambda^{5}+10 \lambda^{6}}{6840} \\
\gamma_{2}=\frac{321 \lambda-510 \lambda^{3}+40 \lambda^{4}+189 \lambda^{5}-40 \lambda^{6}}{6840} & \text { The hybrid (23) becomes, } \\
y_{n+\lambda}=\frac{671}{1216} y_{n+1}+\frac{241}{608} y_{n}+\alpha_{0} y_{n-1}+h^{2}\left(-\frac{503}{4864} f_{n}-\frac{631}{7296} f_{n+1}+\frac{223}{14592} f_{n+2}+-\frac{1}{456} f_{n+3}\right),
\end{array}
$$

with LTE $=\frac{577 y^{(7)}[x] h^{7}}{583680}$. Similarly, for hybrid,

$$
y_{n-\lambda}=a_{2} y_{n+1}+a_{1} y_{n}+a_{0} y_{n-1}+h^{2}\left(b_{0} f_{n}+b_{1} f_{n+1}+b_{2} f_{n+2}+b_{3} f_{n+3}\right) .
$$

We have the following expression for the constants,

$$
\begin{aligned}
& a_{0}=\frac{1}{114}\left(135 \lambda-60 \lambda^{3}+55 \lambda^{4}-18 \lambda^{5}+2 \lambda^{6}\right) \\
& a_{1}=\frac{1}{57}\left(57-78 \lambda+60 \lambda^{3}-55 \lambda^{4}+18 \lambda^{5}-2 \lambda^{6}\right) \\
& a_{2}=\frac{1}{114}\left(21 \lambda-60 \lambda^{3}+55 \lambda^{4}-18 \lambda^{5}+2 \lambda^{6}\right) \\
& b_{0}=\frac{-3313 \lambda+3420 \lambda^{2}+2110 \lambda^{3}-3280 \lambda^{4}+1203 \lambda^{5}-140 \lambda^{6}}{6840} \\
& b_{1}=\frac{-1641 \lambda+1920 \lambda^{3}-50 \lambda^{4}-279 \lambda^{5}+50 \lambda^{6}}{6840} \\
& b_{2}=\frac{321 \lambda-510 \lambda^{3}+40 \lambda^{4}+189 \lambda^{5}-40 \lambda^{6}}{6840} \\
& b_{3}=\frac{-47 \lambda+80 \lambda^{3}-10 \lambda^{4}-33 \lambda^{5}+10 \lambda^{6}}{6840} . \\
& y_{n+1}-2 y_{n}+y_{n-1}=h^{2}\left(\frac{353093 f_{n}}{798336}+\frac{187171\left(f_{n+1}+f_{n-1}\right)}{9979200}-\frac{53\left(f_{n+2}+f_{n-2}\right)}{399168}+\frac{61\left(f_{n+3}+f_{n-3}\right)}{9979200}-\frac{f_{n+4}+f_{n-4}}{4435200}+\frac{40576\left(f_{n-\lambda}+f_{n+\lambda}\right)}{155925}\right) \text {, }
\end{aligned}
$$

with the LTE $=\frac{46507 y^{(14)}[x] h^{14}}{10461394944000}$. The hybrids are,

$$
y_{n+\lambda}=h^{2}\left(\frac{-48103 f_{n}}{552960}-\frac{3323 f_{n+1}}{34560}+\frac{7171 f_{n+2}}{276480}-\frac{43 f_{n+3}}{5760}+\frac{577 f_{n+4}}{552960}\right)+\frac{89 y_{n-1}}{2304}+\frac{487 y_{n}}{1152}+\frac{1241 y_{n+1}}{2304}
$$

with the LTE $=\frac{-157123 y^{(8)}[x] h^{8}}{278691840}$, and

$$
y_{n-\lambda}=h^{2}\left(\frac{-48103 f_{n}}{552960}-\frac{3323 f_{n-1}}{34560}+\frac{7171 f_{n-2}}{276480}-\frac{43 f_{n-3}}{5760}+\frac{577 f_{n-4}}{552960}\right)+\frac{1241 y_{n-1}}{2304}+\frac{487 y_{n}}{1152}+\frac{89 y_{n+1}}{2304},
$$

with the LTE $=\frac{157123 y^{(8)}[x] h^{8}}{278691840}$. Following the analysis like that of (22) on MATHEMATICA v 8 [11], method (27) is thus p-stable.

\section{Implementation of Hybrid SSILMM}

Consider the implementation of the new hybrid methods derived to show the accuracy of these methods in solving some stiff oscillatory and undamped Duffing problems of (1) by resolving the problem of implicitness in the derived hybrid methods. However, methods (22) and (27) are consider for implementation following the ideas in [1] and [6]. Assume that (1) is Lipschitz continuous with reference to $y(x)$ for all $x \in[a, b]$,

$$
\left\|f(x, y)-f\left(x, y^{*}\right)\right\| \leq L\left\|y-y^{*}\right\|,
$$

where $L$ is the Lipschitz constant. The approach of NewtonRaphson iterative method is used to resolve the implicitness in the newly proposed methods. The predictor,

$$
y_{n+2}-2 y_{n+1}+y_{n}=h^{2} f_{n+1}
$$

of order $p=2$ will be used as the starter for the NewtonRaphson iteration with LTE $=\frac{1 y^{(4)}[x] h^{4}}{12}$. The p-stable method,

$$
y_{n+2}-2 y_{n+1}+y_{n}=\frac{h^{2}}{4}\left(f_{n+2}-2 f_{n+1}+f_{n}\right),
$$


is also employ to generate the future solution values $\left\{y_{n \pm j}\right\}_{j=2,3}$ in the case of (22) and $\left\{y_{n \pm j}\right\}_{J=2,3,4}$ in the case of (27) respectively. So that the Newton-Raphson iteration becomes

$$
\begin{gathered}
y_{n+1}^{[t+1]}=y_{n+1}^{[t]}\left(J\left(y_{n+1}^{[t]}\right)\right)^{-1} F\left(y_{n+1}^{[t]}\right), t=0,1, \ldots, w,(3 \\
F\left(y_{n+1}^{[t+1]}\right)=y_{n+1}^{[t]}-2 y_{n}+y_{n-1}=h^{2}\left(\frac{20017}{90720} f_{n}+\frac{67}{362}\right. \\
\text { Example 1: Orbital problem (Source: }[1],[4],[6],[14]) \\
\qquad y^{\prime \prime}+y=0.001 e^{i x}, \\
y(0)=1, y^{\prime}(0)=0.9995 i^{2}=-1,
\end{gathered}
$$$$
F\left(y_{n+1}^{[t+1]}\right)=y_{n+1}^{[t]}-2 y_{n}+y_{n-1}=h^{2}\left(\frac{20017}{90720} f_{n}+\frac{671}{36288}\left(f_{n+1}^{[t]}+f_{n-1}\right)-\frac{241}{2268000}\left(f_{n+2}+f_{n-2}\right)+\frac{13}{4536000}\left(f_{n+3}+f_{n-3}\right)+\right.
$$$$
\left.\frac{18496}{70875}\left(f_{n+\lambda}^{[t]}+f_{n-\lambda}^{[t]}\right)\right) \text {. }
$$

which the theoretical solution is,

$$
y(x)=u(x)+i v(x)=(u(x), v(x)),
$$

where $u(x)=\cos x+0.0005 x \sin x$ and $v(x)=$ $i(\sin x-0.0005 x \cos x)$. The IVP (36) represent motion on a perturbed circular orbit on the complex plane in which the path defined by the point $y(x)=(u(x), v(x))$ spirals slowly where the Jacobian is given by,

$$
J(y)=\frac{\partial F(y)}{\partial y} .
$$

The numerical methods (22) and (27) is applied to solve example $1,2,3$. In the case of the p-stable method in (22),

outward such that its distance from the origin at any given time $x$ is given by,

$$
\Omega(x)=\sqrt{U(x)^{2}+V(x)^{2}},
$$

The interval $0<x \leq 40 \pi$ correspond to 20 orbits of the point $y(x)$,

$$
\Omega\left(x_{f}\right)=\left|y\left(x_{f}\right)\right|=1.00197197653449, x_{f}=40 \pi .
$$

The numerical result is generated using the step size $h=\frac{\pi}{2^{q}}, q=3(1) 13$, and can be seen in Table 1, 2, and 3 .

Table 1. Numerical results of method (22) at $x_{f}=40 \pi$.

\begin{tabular}{llll}
\hline $\mathbf{q}$ & $\mathbf{h}$ & Method (22) $(\Omega)$ & Error $\left|\Omega\left(\boldsymbol{x}_{\boldsymbol{f}}\right)-\Omega\right|$ \\
\hline 3 & $\pi / 2^{3}$ & 1.00203401920494 & $6.20426704474042 \mathrm{e}-005$ \\
4 & $\pi / 2^{4}$ & 1.00200287811217 & $3.09015776829291 \mathrm{e}-005$ \\
5 & $\pi / 2^{5}$ & 1.00198739738256 & $1.54208480669382 \mathrm{e}-005$ \\
6 & $\pi / 2^{6}$ & 1.00197967947316 & $7.70293866714233 \mathrm{e}-006$ \\
7 & $\pi / 2^{7}$ & 1.00197582613246 & $3.84959797039564 \mathrm{e}-006$ \\
8 & $\pi / 2^{8}$ & 1.00197390086563 & $1.92433114087898 \mathrm{e}-006$ \\
9 & $\pi / 2^{9}$ & 1.00197293858310 & $9.62048610331223 \mathrm{e}-007$ \\
10 & $\pi / 2^{10}$ & 1.00197245752955 & $4.80995061558076 \mathrm{e}-007$ \\
11 & $\pi / 2^{11}$ & 1.00197221702471 & $2.40490217517930 \mathrm{e}-007$ \\
12 & $\pi / 2^{12}$ & 1.00197209677777 & $1.20243278001198 \mathrm{e}-007$ \\
13 & $\pi / 2^{13}$ & 1.00197203665567 & $6.01211789241773 \mathrm{e}-008$ \\
\hline
\end{tabular}

Table 2. Numerical results of method (27) at $x_{f}=40 \pi$.

\begin{tabular}{llll}
\hline $\mathbf{q}$ & $\mathbf{h}$ & Method $(\mathbf{2 7})(\Omega)$ & Error $\left|\Omega\left(\boldsymbol{x}_{\boldsymbol{f}}\right)-\Omega\right|$ \\
\hline 3 & $\pi / 2^{3}$ & 1.00203401920494 & $6.20426704474042 \mathrm{e}-005$ \\
4 & $\pi / 2^{4}$ & 1.00200287811217 & $3.09015776829291 \mathrm{e}-005$ \\
5 & $\pi / 2^{5}$ & 1.00198739738256 & $1.54208480669382 \mathrm{e}-005$ \\
6 & $\pi / 2^{6}$ & 1.00197967947316 & $7.70293866714233 \mathrm{e}-006$ \\
7 & $\pi / 2^{7}$ & 1.00197582613246 & $3.84959797039564 \mathrm{e}-006$ \\
8 & $\pi / 2^{8}$ & 1.00197390086563 & $1.92433114087898 \mathrm{e}-006$ \\
9 & $\pi / 2^{9}$ & 1.00197293858310 & $9.62048610331223 \mathrm{e}-007$ \\
10 & $\pi / 2^{10}$ & 1.00197245752955 & $4.80995061558076 \mathrm{e}-007$ \\
11 & $\pi / 2^{11}$ & 1.00197221702471 & $2.40490217517930 \mathrm{e}-007$ \\
12 & $\pi / 2^{12}$ & 1.00197209677777 & $1.20243278001198 \mathrm{e}-007$ \\
13 & $\pi / 2^{13}$ & 1.00197203665567 & $6.01211789241773 \mathrm{e}-008$ \\
\hline
\end{tabular}

Table 3. Numerical results of the hybrid methods when compared with existing methods at $x_{f}=40 \pi$.

\begin{tabular}{lllll}
\hline $\mathbf{q}$ & Lambert \& Watson [14] & Cash [1] & Fatunla [4] & Fatunla et al [6] \\
\hline 3 & 0.965645 & NA & 1.010853 & 1.009580 \\
4 & 0.993734 & 1.004118 & 1.004106 & 1.0096427 \\
5 & 0.999596 & 1.002856 & 1.002502 & 1.006985 \\
6 & NA & 1.002400 & 1.002104 & 1.0048087 \\
7 & NA & NA & 1.002005 & 1.0035590 \\
8 & NA & NA & 1.001980 & 1.0025975 \\
9 & 1.001829 & 1.002057 & 1.001974 & 1.002034 \\
\hline
\end{tabular}


Example 2: Stiff oscillatory IVP (Source: [17])

$$
\begin{gathered}
y^{\prime \prime}(x)+m^{2} y(x)=8 \cos (x)+\frac{2}{3} \cos (3 x), \\
y(0)=1, y^{\prime}(0)=0,
\end{gathered}
$$

where $m=5$. The theoretical solution is,

$$
y(x)=\frac{1}{3}(\cos (x)+\cos (3 x)+\cos (5 x)) .
$$

Where the oscillatory pattern of (40) is generated through the theoretical and numerical solution as in figures 1 and 2 respectively with step size $\frac{\pi}{8}$ at $x=10 \pi$.

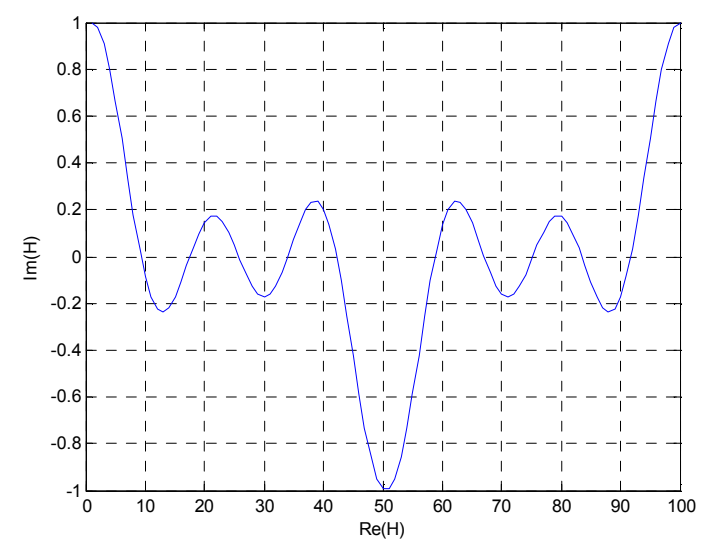

Figure 1. Theoretical solution of IVP (40) over one period.

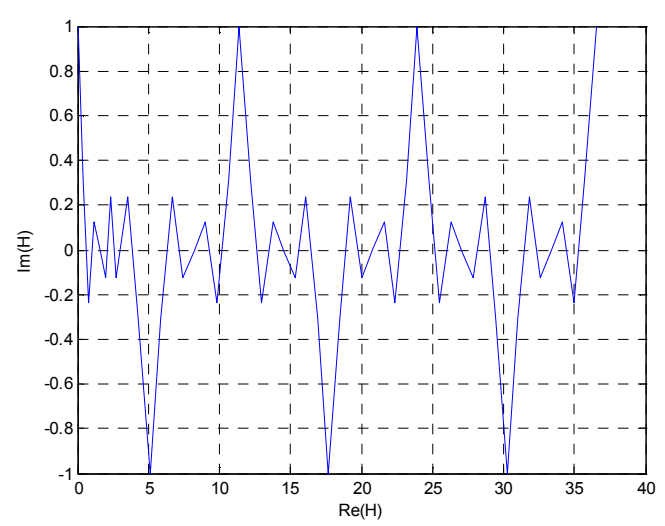

Figure 2. Numerical solution of IVP (40) over five periods.

Example 3: Undamped Duffing IVP (Source: [2], [17], [24]), forced by a harmonic function,

$$
y^{\prime \prime}+y+y^{3}=\delta \cos (\mu x)
$$

with the values of the parameters $\delta=0.002$ and $\mu=1.01$, and with the initial conditions $y(0)=A, y^{\prime}(0)=0$, taking for A the value of the Galerkin approximation $y_{G}$ at $\mathrm{x}=0$. By Urabe's method applied to Galerkin's procedure, [20] has computed the Galerkin's approximation of order $p=9$ to a periodic solution having the same period as the forcing term with a precision $10^{-12}$ of the coefficients of,

$$
y_{G}=\sum_{i=0}^{5} a_{2 i+1} \cos ((2 i+1) \mu x),
$$

$$
y_{G}=\cos (x \mu) a_{1}+\cos (3 x \mu) a_{3}+\cos (5 x \mu) a_{5}+\cos (7 x \mu) a_{7}+\cos (9 x \mu) a_{9}+\cos (11 x \mu) a_{11} \text {, }
$$

where,

$$
\begin{gathered}
a_{1}=0.200179477536, a_{3}=0.246946143 \times 10^{-3}, a_{5}=0.304014 \times 10^{-6}, a_{7}=0.374 \times 10^{-9}, a_{9}= \\
0.460964452 \times 10^{-12}, a_{11}=0.5676 \times 10^{-15} .
\end{gathered}
$$

Where the oscillatory pattern of (42) is generated through the theoretical and numerical solution as in figures 3 and 4 respectively with step size $h=\frac{\pi}{8}$ at $x=40 \pi$.

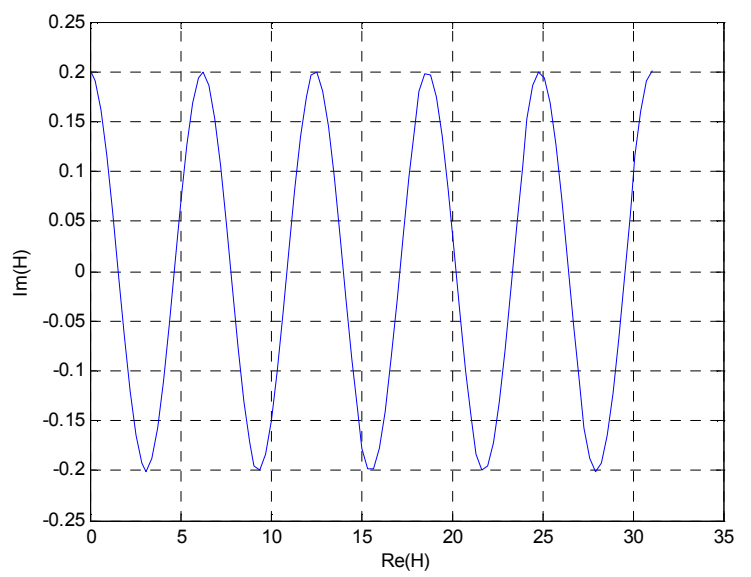

Figure 3. Theoretical solution of IVP (42).

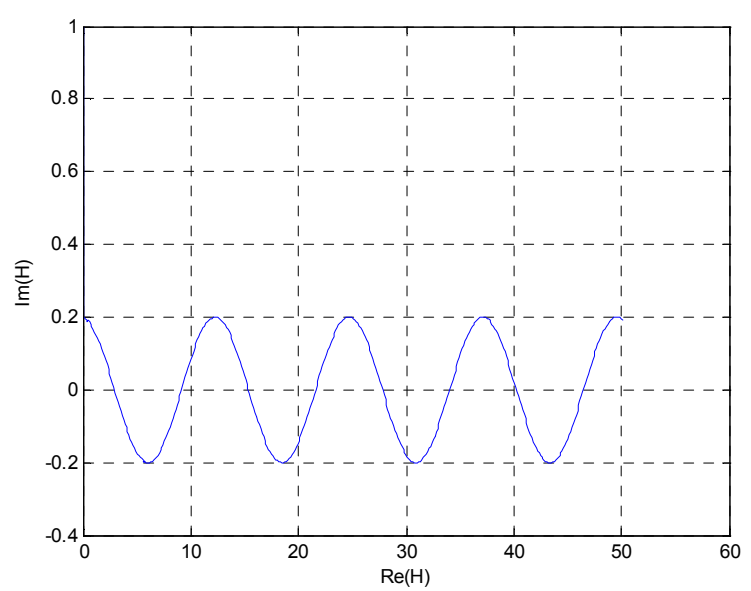

Figure 4. Numerical solution of IVP (42).

\section{Conclusion}

This paper has considered the class of methods defined in 
(16), and p-stable methods based on (16) have been derived. In particular, in (16), p-stable methods were derived with order as high as $p=10$, and 12 which turns to be higher than that of the ones proposed in [16] for the same step length. The order barrier theorem of [3] which has been extended to second-order ODEs by [9] has been bypassed through the use of hybrid methods. The numerical results compare favourably with theoretical and existing results, see tables 1 , 2,3 , and figures $1,2,3$, and 4 as well.

\section{Acknowledgements}

The authors wishes to express their thanks to the founder Professor Francis O. Otunta of the Advanced Research Laboratory in the Department of Mathematics, University of Benin, Benin City, Nigeria where the analysis and the initial draft of this work was carried out.

The second author is indeed grateful to the founder Professor Neil. Turok of African Institute for Mathematical Sciences, Kigali City, Rwanda for providing a conducive computing environment for the first author which enabled him to complete the remaining part of the analysis of this work and the final draft.

The authors are grateful to the referees for their careful reading of this manuscript and useful comments.

\section{References}

[1] Cash J. R., (1981), High order P-stable Formulae for the Numerical Integration of Periodic Initial Value Problems. $J$. Numer. Maths., 37: 355-370.

[2] Chawla M. M., and Neta B., (1986), Families of two-step fourth order P-stable methods for second order differential equations, J. Comp. and Appl. Maths 15: 213-223.

[3] Dahlquist, G., (1978), On Accuracy and Unconditional Stability of the Linear Methods for Second Order Differential Equations, BIT, 18: 133- 136.

[4] Fatunla S. O., (1985), One-leg Hybrid Formula for Second Order IVPs. Computers and Mathematics with Applications, 10: 329-333.

[5] Fatunla S. O., (1988), Numerical Methods for Initial Value Problems in Ordinary Differential Equations, Academic Press, Boston, USA.

[6] Fatunla S. O., Ikhile M. N. O., and Otunta F. O., (1997), A Class of P-stable Linear Multistep Num. Methods. Int. J of Comp Math. 72: 1-13.

[7] Fukushima T., (1998), "Symmetric multistep methods, revisited", In Prec. of the 30 tu Syrup. on Celestial Mechanics, 4-6 March 1998, Hayama, Kanagawa, Japan (Edited by T. Fukushima, T. Ito, T. Fuse and H. Umehara): 229-247.

[8] Fukushima T., (1999), Super-implicit multi-step methods, Proc. of the 31th Symp. on Celestial Mechanics, Kashima Space Research Center, Ibaraki, Japan (Edited by H. Umehara): 343-366.
[9] Hairer E., (1979), Unconditionally Stable Methods for Second order Differential Equations, Numerical Maths., 32: 373 - 379.

[10] Ibrahim O. M. (2016), High Order Symmetric Super-Implicit Hybrid LMM with Minimal Phase-Lag Error. M. Sc. Thesis, University of Benin, Nigeria.

[11] Borwein J. M., and Skerritt M. P., (2012), An Introduction to Modern Mathematical Computing with Mathematica, Springer.

[12] Jain M. K., Jain R. K., and Anantha Krishnaiah U., (1979), Pstable Methods for Periodic Initial Value Problems of Second Order Differential Equations. BIT 19: 347-355.

[13] Lambert J. D., (1973), Numerical Methods for Ordinary Differential Systems: the Initial Value Problem. John Wiley \& Sons Ltd.

[14] Lambert J. D., and Watson I., (1976), Symmetric Multistep Methods For Periodic Initial Value Problems, J. Inst. Math. Appls., 18: 189-202.

[15] Mehdizadeh Khalsaraei 1 M. and Molayi M., (2015) P-Stable Hybrid Super-Implicit Methods for Periodic Initial Value Problems. Journal of mathematics and computer science. 15: $129-136$.

[16] Neta B., (2005), P-stable Symmetric Super-Implicit Methods for Periodic Initial Value Problems, Comput. Math. Appl. 50: 701-705.

[17] Neta B. (2007), P-Stable High-Order Super-Implicit and Obrechkoff Methods for Periodic Initial Value Problems, Computers and Mathematics with Applications 54: 117-126.

[18] Otto S. R. and Denier J. P., (2005), An Introduction to Programming and Numerical methods in MATLAB, SpringerVerlag, London.

[19] Simos T. E., (1993), A P-stable Complete in Phase Obrechkoff Trigonometric Fitted Method for Periodic Initial-value Problems, Prec. Royal See. London A, 441: 283-289.

[20] Van Dooren R., (1974), Stabilization of Cowell's classical finite difference methods for numerical integration. $J$. Comput. Phys. 16: 186-192.

[21] Okuonghae R. I., and Ikhile M. N. O., (2014), A-Stable High Order Hybrid Linear Multistep Methods for Stiff Problems, $J$. Algor. Comp. Technol., 8 (4): 441-469.

[22] Okuonghae R. I., and Ikhile M. N. O., (2015), Stiffly Stable Second Derivative Linear Multistep Methods with two Hybrid Points, Num. Analys. and Appl., 8 (3): 248-259.

[23] Shoki A., and Saadat H., (2015), High Phase-Lag Order Trigonometrically Fitted Two-Step Obrechkoff Methods for the Numerical Solution of Periodic IVPs Num. Algorithm, 68 (2): $337-354$.

[24] Shoki A., (2017), A New High Order Implicit Four-Step Method with Vanished Phase-Lag and some of its Derivatives for the Numerical Solution of the Radial schrödinger equation, J. Modern Methods in Num. Maths., 8 (1-2): 1-16.

[25] Ibrahim O. M., (2017), On the construction of high accuracy symmetric super-implicit hybrid formulas with phase-lag properties, Accepted for publication: Transaction J. of Nigeria Ass. of Mathematical Physics. 